\title{
Aciclovir en el tratamiento de la parálisis facial idiopática
}

\author{
A. Rodríguez Blanco, J. Morera Montes ${ }^{1}$ \\ Residente $3^{\circ}$ año de MFyC. ${ }^{1}$ Especialista en Medicina Familiar y Comunitaria. \\ Centro de Salud V Centenario. San Sebastián de los Reyes, Madrid
}

\section{RESUMEN}

Recientemente se ha propuesto la utilización de aciclovir en el tratamiento de la parálisis facial idiopática, debido al auge que la hipótesis vírica ha tenido en los últimos años.

Varios ensayos clínicos han intentado demostrar la utilidad del uso combinado de aciclovir y prednisona frente al uso de prednisona sola.

Revisamos la literatura con el fin de establecer pautas de actuación en Atención Primaria y concluimos que con los datos disponibles en la actualidad no existe evidencia suficiente para recomendar el uso de aciclovir en el tratamiento de la parálisis facial idiopática.

Palabras clave: Parálisis facial idiopática. Aciclovir. Prednisona. Tratamiento.
Acyclovir in idiopatic facial paralysis treatment

\section{ABSTRACT}

Recently, acyclovir use has been proposed for the treatment of idiopatic acute paralysis due to the hypothetical viral origin of the disease.

Several trials has tried to conclude that acyclovir-prednisona is superior to prednisone alone.

We review the latest studies to define protocols in Primary Health Care for the medical treatment of idiopatic facial paralisis but current evidence does not support the recomendation that acyclovir should be use in this disorder.

Key words: Idiopatic acute paralysis. Acyclovir. Prednisone. Treatment.

\section{PREGUNTA ESTRUCTURADA}

¿Está indicada la utilización de aciclovir en la parálisis facial idiopática?

La parálisis facial idiopática es una entidad que afecta aproximadamente a 20 personas por 100.000 habitantes. Alrededor del $70 \%$ de los pacientes afectados se recuperan satisfactoriamente sin tratamiento, siendo necesarios entre 12 y 18 meses tras el inicio del cuadro para poder valorar la existencia o no de secuelas a largo plazo ${ }^{1}$.

Desde que se describió por primera vez la parálisis facial en 1830, múltiples teorías han intentado explicar su etiopatogenia, entre las que se encuentran la hipótesis de isquemia vascular, inmunológica y de compresión aunque en los últimos años ha surgido la hipótesis vírica que considera que esta entidad puede deberse a una reactivación del virus 
herpes simple acantonado en el nervio facial. Debido a ello se postula en los últimos años el uso de fármacos antiviriásicos (aciclovir) en el tratamiento de esta enfermedad ${ }^{2}$.

\section{La búsqueda}

Para responder a nuestra duda tratamos de encontrar una buena revisión sistemática, a la vez que analizamos los ensayos clínicos publicados hasta el momento con el fin de determinar la mejor respuesta disponible. En ningún caso se trata de un metaanálisis, sino de una descripción de los trabajos publicados hasta el momento que puedan arrojar novedades en el tratamiento de la parálisis facial idiopática también conocida como parálisis de Bell.

Realizamos la búsqueda utilizando los siguientes términos: facial paralysis (MESH) AND acyclovir (MESH) AND Randomized Controlled Trial (PT) OR Treatment (WORD). Limitis: All ages: $19+$ years, only items with abstracts, gender, human. Realizada en Medline, Embase y Lilacs en diciembre del 2002.

\section{El estudio}

Siguiendo los criterios anteriormente definidos seleccionamos el presente estudio por tratarse de una revisión sistemática que incluye y evalúa los trabajos encontrados en nuestra búsqueda ${ }^{3,4}$. El estudio seleccionado es el siguiente: Aciclovir for Bell's palsy (idiopatic facial paralysis).

Los autores hicieron una búsqueda en bases de datos del registro del Grupo de Enfermedades Neuromusculares Cochrane, Medline, Embase y Lilacs, y contactaron con los autores de los distintos ensayos clínicos encontrados.

Se seleccionaron los ensayos clínicos randomizados o cuasi-randomizados que usaban aciclovir solo o en combinación con otros fármacos en pacientes con parálisis facial idiopática.

Se identificaron un total de 4 ensayos clínicos randomizados y no se obtuvieron datos adicionales tras contactar con los autores de los mismos. De los 4 estudios analizados solamente 2 cumplían los criterios de inclusión y recogían resultados de al menos 200 pacientes, (el número final de pacientes que entraron en el estudio estadístico fue de 99 en el trabajo de Adour y 101 en el trabajo de De Diego) ${ }^{1}$; uno de estos ensayos evaluaba el uso de aciclovir en combinación con corticoides frente a corticoides solos; mientras que el otro sólo aciclovir versus corticoides.

En cuanto a los resultados no existen datos so- bre la proporción de pacientes con una recuperación incompleta al año de iniciarse la parálisis facial; sin embargo el análisis de los datos se realizó tras la finalización del estudio que fue a los $3^{1}$ y 4 meses $^{4}$ de inicio de tratamiento con aciclovir (éste último consideró que era el mejor momento para finalizar el estudio debido a "la amplia experiencia que tienen en el tratamiento de la parálisis facial").

Se obtuvo un resultado estadísticamente significativo en el grupo tratado con aciclovir en el trabajo de Adour ${ }^{4}$. No se evaluó la aparición de efectos adversos en ningún trabajo.

Sólo un paciente presentó parálisis facial completa a los 6 meses de empezar el tratamiento', fue asignado al grupo control y el grado de recuperación alcanzado posteriormente no se menciona. Otras secuelas como las sincinesias motoras y las "lágrimas de cocodrilo" no son evaluadas al año de producirse la parálisis facial existiendo datos únicamente a los 4 meses del inicio de ésta.

Finalmente sólo un estudio muestra diferencias estadísticamente significativas entre los grupos de tratamiento a favor del grupo que utilizó aciclovir.

Queremos destacar datos encontrados en nuestra búsqueda y que no son mencionados en la revisión hecha por el centro Cochrane, en primer lugar un trabajo publicado en 1999 por Ibarrondo et $\mathrm{al}^{6}$, en el que se estudian 300 pacientes con parálisis facial de Bell distribuidos en 3 grupos a los que se administró aciclovir, corticoides o complejos vitamínicos del grupo B. Todos los pacientes se evaluaron al inicio de la parálisis, a los diez días, al mes, tres, seis, nueve y 12 meses mediante exploración ORL-otoneurofacial. Tras el análisis estadístico se llegó a la conclusión de que la evolución de estos pacientes es independiente del tipo de tratamiento recibido. El estudio no es adecuado por su diseño para evaluar tratamientos, ya que no es controlado, randomizado ni doble ciego, aunque el tiempo de seguimiento en este estudio sí es el recomendado por la Cochrane.

Todos los trabajos que revisamos evaluaron los pacientes según la clasificación clínica de House y Brackmann, que presenta un cierto número de imprecisiones al ser una apreciación subjetiva, en particular en relación a las sincinesias, con lo que los resultados pueden introducir el sesgo del observador.

Las conclusiones que se derivan del análisis ponen de manifiesto la necesidad de realizar más ensayos clínicos controlados, randomizados, y doble ciego durante un periodo de estudio de al menos 12 meses de duración para poder recomendar el aciclovir de forma rutinaria en el tratamiento de la parálisis facial idiopática. 
Tabla I

USO DE ACICLOVIR EN EL TRATAMIENTO DE LA PARÁLISIS FACIAL IDIOPÁTICA

\begin{tabular}{lcccc}
\hline Estudio & Número pacientes & Tratamiento & SE & T. seguimiento \\
\hline Ramos et al, 1992 & 45 & Az+C vs C & NO & 2 meses \\
Adour et al, 1996 & 119 & Az+C vs C & SI & NO \\
De Diego et al, 1998 & 101 & Az vs C & NO & 12 meses \\
Ibarrondo et al, 1999 & 300 & Az/ C/ Vit & Nes \\
\hline Az: aciclovir; C: corticoides; Vit: vitaminas grupo B1. SE: Significación estadística $(p<0,05) ;$ : tiempo.
\end{tabular}

Az: aciclovir; C: corticoides; Vit: vitaminas grupo B1. SE: Significación estadística ( $p<0,05)$; T: tiempo.

\section{COMENTARIOS}

Se trata de una revisión sistemática en la que el objeto de estudio está claramente definido, así como los criterios de selección de los distintos ensayos clínicos analizados, teniendo en cuenta para su aceptación tanto el número de pacientes, el tipo de ensayo clínico (randomizado o cuasi-randomizado) el uso de aciclovir como tratamiento de la parálisis facial, la significación estadística de los resultados y el tiempo de seguimiento, dato fundamental para sacar conclusiones ya que el tiempo necesario para dar por finalizada la recuperación tras un episodio de parálisis facial oscila entre 12 y 18 meses, como se ha comentado anteriormente.

De los estudios incluidos el de Ramos et al está bien diseñado, aunque no encuentran una diferencia estadísticamente significativa entre el tratamiento con aciclovir y aciclovir más corticoides; el de Adour et $\mathrm{al}^{4}$, está impecablemente diseñado siendo prospectivo, controlado, aleatorizado y doble ciego, utiliza un número de pacientes adecuado y establece diferencias significativas aunque la diferencia de recuperación entre los dos grupos está en el límite de la significatividad ( $p=0,02$ a 0,06 ). A pesar de su buen diseño y método hay que añadir que se evaluaron los pacientes sólo a los cuatro meses de evolución. Por ello puede que las diferencias no sean debidas a la eficacia del tratamiento, lo que hace que las conclusiones carezcan de la validez suficiente ${ }^{6}$.

El resto de las variables analizadas en los distintos estudios aparecen reflejadas en la tabla I (se incluyen los 4 ensayos clínicos identificados inicialmente) $)^{1,3,4,6}$.

\section{CONCLUSIONES}

Actualmente no existe evidencia suficiente para recomendar el uso de aciclovir en el tratamiento de la parálisis facial idiopática en Atención Primaria, si bien próximos estudios pueden añadir una nueva posibilidad en el arsenal terapéutico de la parálisis facial idiopática.

\section{CORRESPONDENCIA:}

A. Rodríguez Blanco

Centro de Salud V Centenario

C/ Real, 91

28100 San Sebastián de los Reyes (Madrid)

\section{Bibliografía}

1. De Diego JI, Prim MP, De Sarriá MJ, Madero R, Gavilán J. Idiopathic facial paralysis: a randomized, prospective, and controlled study using single-dose prednisone versus acyclovir three times daily. Laryngoscope 1998; 108 (4 Pt 1): 573-5.

2. De Diego JI, Prim MP, Gavilán J. Etiopatogenia de la parálisis facial periférica o de Bell. Rev Neurol 2001; 32 (11): $1055-9$.

3. Ramos Macías A, de Miguel Martínez I, Martín Sánchez AM, Gómez González JL, Martín Galán A. Incorporación del aciclovir en el tratamiento de la parálisis periférica. Un estudio en 45 casos. Acta Otorrinolaring Esp 1992;4 3, (2): 117-20.

4. Adour K, Ruboyianes JM, Von Doersten PG, Byl FM, Trent CS, Quesenberry Jr CP, et al. Bell's palsy treatment with acy- clovir and prednisone compared with prednisone alone: a doble-blinde, randomize contolled trial. Ann Otol Rhinol Laryngol 1996; 105 (5): 371-8.

5. Sipe J, Dunn L. Aciclovir for Bell's palsy (idiopatic facial paralysis). Cochrane Database Syst Rev 2001; (2): CD001869.

6. Ibarrondo J, Navarrete ML, Encarnación LF, Quesada P, Crespo F, García M, et al. Treatment of idiopatic facial paralysis: corticoids versus acyclovir versus empirical treatment. Acta Otorrinolaringol Esp 1999; 50 (2): 118-20.

7. Salinas R. Parálisis de Bell (parálisis facial periférica) En: Bonfill X, eds. Evidencia Clínica. Bogotá, Colombia: Editorial Legis. 2002. p. 1153-7. 